\title{
Energy Holes Minimization with Enhanced AEIAWSNHP Algorithm
}

\author{
Chinmaya Kumar Nayak, Satyabrata Das
}

\begin{abstract}
The developments of wireless sensor network are motivated by many applications. It needs the Sensor nodes location. Sensor nodes are based primarily for identification procedure to resolve their significant position. In general, Sensor nodes are capable of some restricted power supply. As a result for detecting the power of sensor nodes an Identification algorithm is used by wireless sensor network. An Efficient Identify Algorithm for Wireless Sensor Networks with High Precession (AEIAWSNHP) is one efficient energy identification algorithm that has been proposed recently. In this work we examine the blow of using three techniques through the improvement of AEIAWSNHP in civilizing the energy efficient of enhanced AEIAWSNHP.At first, a Distinct-assessment Method, where a node estimate its location simply at one time. Secondly, active power manages; in this place the mention nodes decrease their communication power according to the gap to the node that transmits the position requirements. Third, an addition and expanding request speed method, that regulate the frequentness of dispatching the locate inquiry. The simulation result present that the new technique decreases the power utilization of the updated AEIAWSNH, Accuracy of the location assessment remains unchanged.
\end{abstract}

Keywords : Identify procedure; power competence; enhanced AEIAWSNHP; consecutive-enhancement identify algorithm; Wireless sensor network.

\section{INTRODUCTION}

With the explosion of wireless technology and merging of communication has led to the growth of wireless sensor networks (WSN) with a huge number of low-cost sensors such as computers and communications, storage and power supplies [1]. These sensor nodes can detect, determine in addition to gather unprocessed information beginning the surroundings, Carry out easy calculations as well as transfer just the necessary with partly performed information absolutely junction node [2][3].

WSN is widely used in various industries and has been used in many smart real life uses [5] - [8]. For the most of the uses need with the intension of node locations are detected. In addition, different WSN technologies require very accurate information about a node such as set of connections organization with direction-finding set of rules for transfer.

\section{Revised Manuscript Received on December 30, 2019.}

* Correspondence Author

Chinmya Kumar Nayak*, Department of Computer Sc. \& Engineering, Veer Surendra Sai University of Technology (VSSUT), Burla, Odisha, India.

Satyabrata Das, Department of Information Technology, Veer Surendra Sai University of Technology (VSSUT), Burla, Odisha, India.

(C) The Authors. Published by Blue Eyes Intelligence Engineering and Sciences Publication (BEIESP). This is an open access article under the CC BY-NC-ND license (http://creativecommons.org/licenses/by-nc-nd/4.0/)
Main benefits of localization of data include improving WSN efficiency, identifying the location, facilitating multiple application services, and assisting many features on the network system [8].

Networked storage networks are widely used in various fields and have been utilized various real life uses for Smart environment [5] - [8].All most all of these techniques need that the place of the nodes to be reached a decision. In addition, various WSN techniques require a very precise knowledge of the position in junction, such as: Such as, set of connection organization protocol in addition to reinstallation direction finding. Key benefits of trunk data is for improving the competence of WSN network, Recognize position of the occurrence, enabling multiple application services, and supporting multiple systems.

All most all sensor nodes batteries are not lasting long, which sometimes cannot be reconstructed. Therefore, the lifetime of the detection nodes depends mainly on the battery life [10][11]. For that reason, power-sensitive localization procedure, such as [13] - [14], must employ various methods to decrease the process of computing and transmission overload, thus decreasing power uses [2], [9].

To connect the GPS handset for each sensing node is the choice for determine a position of the node. Though, the decision increases the price in addition to complication of node. In addition, this lead to enhance the power utilization as GPS is with on one communications strategy that consumes a lot of energy. Therefore, the devices of all GPS receiver nodes are not practical solutions. Convenient location adjustment is a suitable way so as to use on fixed WSN. This situation significantly enhances operating overheads.

Identification procedure can be treated as an appropriate result for identifying the position of the sensor nodes. Rather connecting a GPS handset on each node, some of the sensor nodes resolve supplies a GPS receiver. These nodes referred to as "markers".Initially, other nodes unknown about their position and are so named "unknowns". "Unknown" utilizes the location procedure to assess the location, and this position becomes "known". So, word "reference" in literature uses reference for "markers" as well as other "well-known", which contribute to the evaluation of the "unknown" positions.

In the article of [16] the author presented AEIAWSNHP algorithm. AEIAWSNHP is more efficient that other algorithms. Several experiments and an exhaustive comparison with several identification algorithms were carried out. The main purpose of this article is to improve the energy efficiency using AEIAWSNHP algorithm.

The remaining part is maintained as given: Segment 2 explains the enhancement of the AEIAWSNHP procedure in addition to focuses with important ideas in this procedure for power competence.

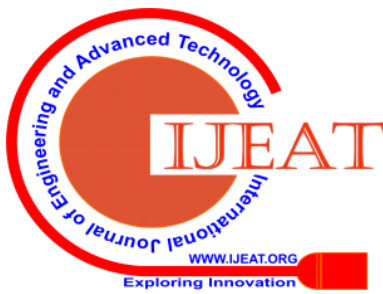




\section{Energy Holes Minimization with Enhanced AEIAWSNHP Algorithm}

Segment 3 explains the methods which are used to increase the energy efficiency of enhanced AEIAWSNHP, namely the assessment procedure, Active power management and Improvement and rapid growth order rate. Network simulators NS2 utilize to evaluate the efficiency of existing procedure in addition to enhanced AEIAWSNHP. Segment4 evaluates the outcomes. Segment5 is the conclusion.

\section{ENHANCED AEIAWSNHP PROCEDURE}

Main purpose of the development of the enhanced AEIAWSNHP procedure is the get better result of identification procedure by increasing accuracy and robustness of the location assessment. The reduction of consumption of energy [16] and the establishment of a safety procedure in opposition to hateful nodes that challenge the identification method [17].The accuracy of location assessment is the main focus of location method. The Enhanced AEIAWSNHP procedure plays a major role to overcome these problems of accuracy of location assessment.

i. Initialization

If(Final=true) then exit

Broadcast "location request" messages

Receive "location response" messages from neighbouring reference (Ri)

If $(\mathrm{C}(\mathrm{Ri})<3$ then exit.

ii. First location assessment

Select a subset of references $\mathrm{Si}$ from $\mathrm{Ri}$

Measure distance to the references in $\mathrm{Si}$

Apply MMSE to determine an initial position $\hat{z}_{i}$

iii. Advanced location assessment

$$
\begin{aligned}
& \text { for }(\mathrm{j}=1 \text { to } \mathrm{C}(\mathrm{Si})) \\
& \hat{e}_{i j}^{d}=\left|\left\|\hat{Z}_{i}{ }^{0} \cdot \hat{z}_{j}\right\| \cdot \hat{d}_{i j}\right| \\
& \operatorname{If}\left(\hat{e_{i j}^{d}}>e_{\max }^{d}\right) \text { then }(\text { enhancement }=\text { true }) ; \text { break. } \\
& \text { If(enhancement=true) } \\
& \text { For(j=1 to } C(R i)) \\
& \hat{e}_{V}^{d}=\left|\left\|\hat{z}_{i}^{0} \cdot \hat{z}_{j}\right\| \cdot \hat{d}_{i j}\right| \\
& \text { If }\left(\hat{e}_{V}^{d}>\hat{e}_{\text {max }}^{d}\right) \text { then eliminate } \mathrm{r}_{\mathrm{j}} \\
& \text { Estimate refined position as } \hat{z_{i}} \text { shown in } 2 \\
& \text { Else } \\
& \hat{z_{i}}=\hat{z}_{i}^{0} \\
& \text { iv. Location revise } \\
& D_{a u e}=\sum|| \hat{z}_{k} \cdot z_{j}|| \cdot \hat{d}_{i l} \mid \\
& \text { If }\left(D_{\text {asc }}^{k}<D_{\text {acc }}^{k \cdot 1}\right) \\
& \hat{z}_{i} \text { Will be accepted } \\
& \text { If }\left(D_{a c c}<T_{a c c}\right) \text { then }(\text { final }=\text { true })
\end{aligned}
$$

A node transmits a "position solicitation" packet to start the process of position assessment. Neighbor indication reply with their position data. A new intelligent indication choice technique represent in Fig. 1 will choose a division of accessible references, in which priority is not given to the references with high potential of position estimation. To approximate the start position the node will use the particular reference. To get better the judgment of the location, the location the node filter a few of the reference use in the primary step, except this time removing references with a long space assessment fault and then returns to approximate its location. This estimation is acceptable by the node if the accuracy is better than the previous estimation. At last node verify during future iteration whether it is feasible to increase the accuracy of the assessment of the location of the node; if not then the current assessment is considered as final and transfer of location demand will be stop.

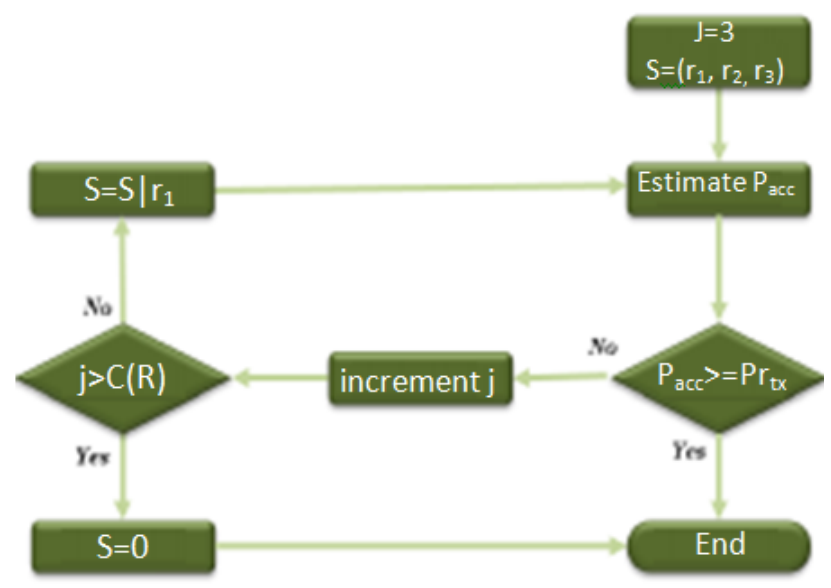

Fig. 1.Elegant position choice process [16]

Enhanced AEIAWSNHP procedure uses numerous methods to improve the power competence. Such as:

- The reply method reduce the rate of communication by means of dropping the amount of reply packet, just reference through a definite stage of correctness distributing reply packet to the position demand. It decreases computational costs with minimizing amount of repetitions.

- The intelligent position choice technique decreases the calculation rate due to its cleanness and uses of the least amount of reference feasible. This technique finds the estimation instance with no additional broadcast communication among the nodes, which reduces message costs.

- The principles of termination reduce computational along with message costs through dropping the amount of iterations essential to obtain a perfect estimate of location. The nodes surrounding source through elevated positional correctness (eg, tags that have a higher positional accuracy than known marks) require fewer iterations before reaching an acceptable positional accuracy level. Exclusive of extinction criterion, these nodes will continue to re-estimate their location exclusive of civilizing their correctness and wasting their power.

This article purpose is to assess the blow by means of different techniques to progress the power competence of the improved AEIAWSNHP procedure. For that reason additional particular on the improved AEIAWSNHP procedure, the different methods measured by improved AEIAWSNHP and the outcome assessment of the procedure [16].

\section{ADDITIONAL POWER COMPETENCES}

Three different techniques are examined in this AEIAWSNHP algorithm to get better result. These techniques are as follows: 


\section{i. Distinct-assessment Method}

The method "different evaluation" method [15][16] is very simple method use for the identification procedure. Sensor nodes approximate its location one time in this technique. When this station receives position data as a minimum from three instance of, it estimates its location and stops localization method. Operation of this technique is very easy, do not need close criterion, needs repetitions and is extremely power competent. Though, previous motto of identification schemes, for example the correctness of the evaluation, may not be achieved. A few try to enhance the correctness of location procedure; however it possibly will boost the complication of this procedure [17]. In addition, few of this procedure are able to just work in positive situation. Such as, the procedure planned in [18] needs a triangular assignment of the labels in positive places.

Estimating location of the node just at a time could not give better correctness, particularly in clamour surroundings. Consequently, A number of localization procedures, such as [22], [23], go after one more method. With this technique, to enhance the accuracy of the location assessment, the sensor node treats its place a number of periods. On the other hand this technique enhances computing and transmission slides, thus increasing power utilization. The most important elements of identification procedure on the technique are stop criterion; its vital responsibility is to enhance the power competence of identification algorithms.

Improved AEIAWSNHP is of next category; that is, a sequential modification method, anywhere node iterates the estimate for improve positioning correctness. With the assumption of solitary assessment method, locations bring up to date determination uncared for the fourth step. so the node assess this location just one time.
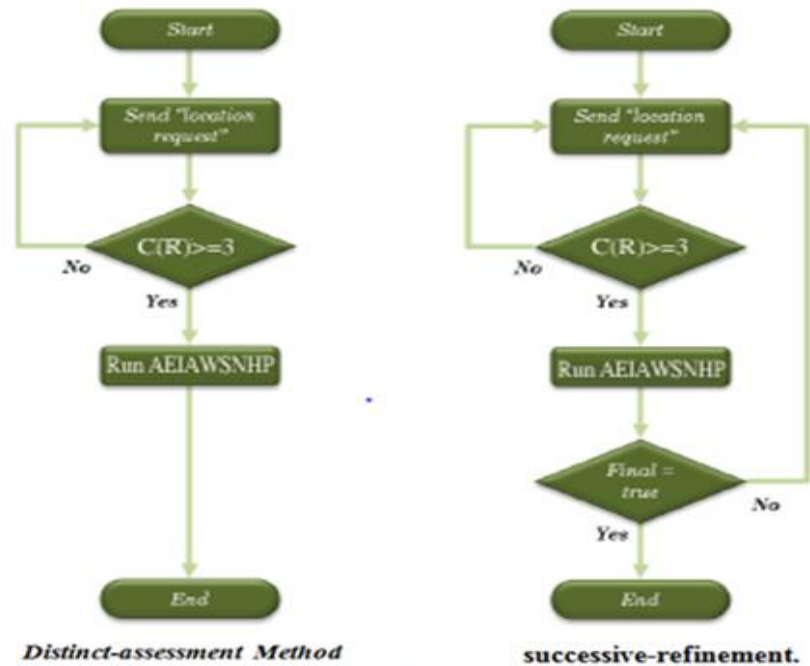

Fig.2.Distinct-assessment and consecutive modification technique.

In general terms, in distinct assessment method, while a node receive position data larger in comparison to least amount of desired mention of, simply it assess this location. With enhanced AEIAWSNHP procedure, it is not just one situation to assess this location for the node. The intelligent mention choice process; choose the best most excellent and exact subdivision remark, result accurate existing remark.

\section{ii. Active control manage}

Main objective of active control manage method to flexible alter the stage of communication control build with the space among the communicating and collecting nodes. as an alternative ongoing transmit the package of data by means of highest communication control extend over the whole area, Each sensor node actively mention desired communication control to make possible the package of data get to the target[4].

Active energy manages method be able to utilize with the enhanced AEIAWSNHP procedure it represent: node utilize highest communication energy transmission "position demand" package for data, guarantee that nodes surrounded by communication area collect package of data. Who will collect the call assesses the gap with solicit node by applying equation (1).It also approximate desired communication energy to permit the "position reply" package of data to arrive at the solicit sensor node [4].

$d=\sqrt{\frac{P_{t} \times G_{t} \times G_{r}}{P_{r}}} \times \frac{\lambda}{4 \times \pi}$

Here $\mathrm{d}$ represent gap among the neighbor node and solicit node, Pt represent communication control, $\mathrm{Pr}$ represent collect control, Gt represent communication transmitter grow, Gr represent recipient transmitter grow and $\lambda$ represent wavelength of the signal.

\section{iii. Progressive and Expanding Solicitation speed}

The main objectives of localization method to minimize the amount of beacon. Primarily, the close by unidentified nodes regulates the location. As a result, the unidentified nodes distant starting the beacons requires additional time to choose its location compare by means of another which is nearer to it. So, with the method, the solicitation speed $\left(\Delta t_{\text {req }}\right)$ is restructured following every repetition; by means of progressive or expanding. If the node calculates its location in the specified repetition or updated the correctness of recent location, the requesting rate will be incremented by $1\left(\Delta t_{\text {req }}=\Delta t_{\text {req }}+1\right)$, or else update the requesting rate by multiplying with $2\left(\Delta t_{\text {req }}=2 * \Delta t_{\text {req }}\right)$.

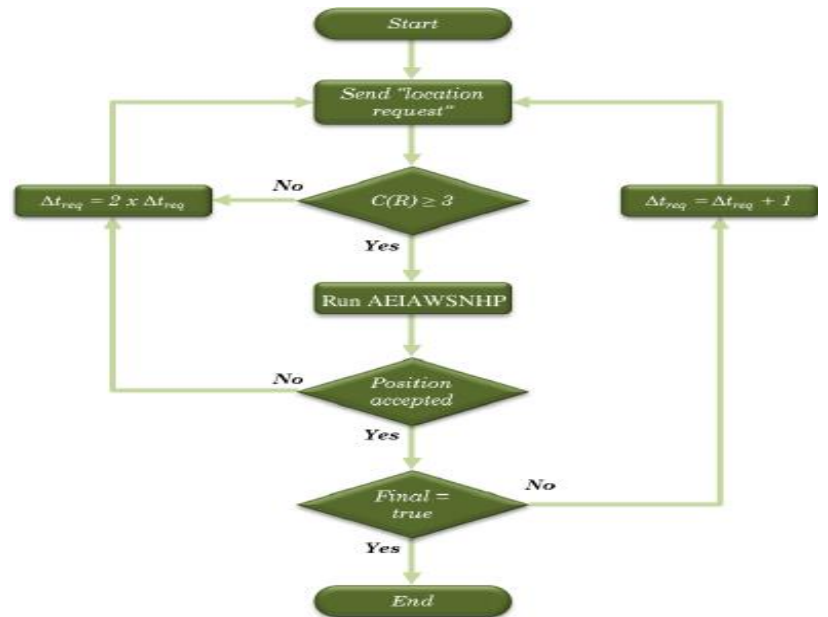

Fig. 3. Progressive and expanding solicitation speed technique

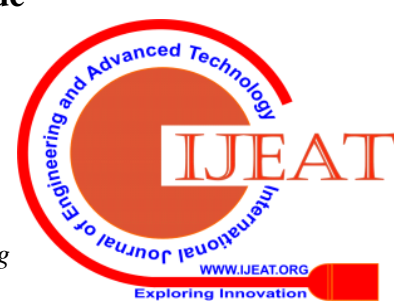




\section{Energy Holes Minimization with Enhanced AEIAWSNHP Algorithm}

\section{EXPERIMENT OUTCOMES}

Mainly NS2 network simulator is used in the study to assess different procedure, methods and protocols of wireless sensor networks. Though, the main NS2 not provide simplest way to execute localization procedures. The authors of [21][22] provided the open source to permit the experiment of wireless sensor network localization procedure. So, we preferred NS2 to execute the proposed algorithm .In our experiment we choose 78 nodes randomly deployed in an area of 300m X 300m.from the 78 nodes 13 are beacons and the remaining not aware about their locations.

Originally, enhanced AEIAWSNHP's output is contrast by means of 2 more localization procedure, one is distinct and another one is consecutive. Distinct go for distinct-assessment technique at the same time as Consecutive go for the consecutive-assessment technique. Finally we evaluate the performance and correctness of enhanced AEIAWSNHP procedure.

Efficiency of the procedures is calculated by two key approaches: firstly localization fault, it returns by means of position correctness. Average fault is calculated time to time as represent in equation (2). Cause for evaluating the average fault used ratio of communication gap to distinct from other implementation.

Average fault $t_{t}=\left(\frac{1}{n} \sum_{i=1}^{n}\left\|\hat{z}_{i}-z_{i}\right\|\right) \frac{1}{r_{t x}} 100 \%$

Here, $n$ represents the cumulativeness at a particular time $\mathrm{t}, \mathrm{z}$ represents genuine node position and the symbol $\hat{Z}$ represent predictable location by means of the localization procedure. Next approach is power utilization by means of transmission.

\section{i. Distinct-assessment Method}

Fig. 4 represent improve the power competence of the existing procedure. Conversely, the average fault is a little high in comparison to existing procedure, as represent in Fig. 8. Represent assessment of the node location just one time. Though, at rest output the chance of correctness of the existing reference.

In a disturb surroundings the space dimension fault determination be high, that enhance the average fault of the location assessment. So, in disturb surrounding it is not appropriate to utilize distinct-assessment method, particularly if correctness is a serious matter. For betterment of correctness of the location assessment in such a surroundings, localization procedures are supposed to consider the consecutive-modification method [17].

\section{ii. Active Energy manage}

Fig. 4 represent by the method of Enhanced AEIAWSNHP do not improve the power competence extremely a lot due to various cause. Main cause is the methods utilize simply to modify the depth of power for transmitting the "position reply" packet, at the same time as the nodes utilize utmost communication power to transmit the "position reply" packets. Enhanced AEIAWSNHP drop the amount of "position reply" packets, so this method will not create any impact on power competence of the Enhanced AEIAWSNHP procedure.

\section{iii. Progressive and Expanding Requesting rate}

In the Fig. 4, Enhanced AEIAWSNHP, while with the progressive and expanding requesting rate method, ingest a smaller amount power than the existing algorithm .Fig. 5 represent with the intention of correctness is somewhat improved.

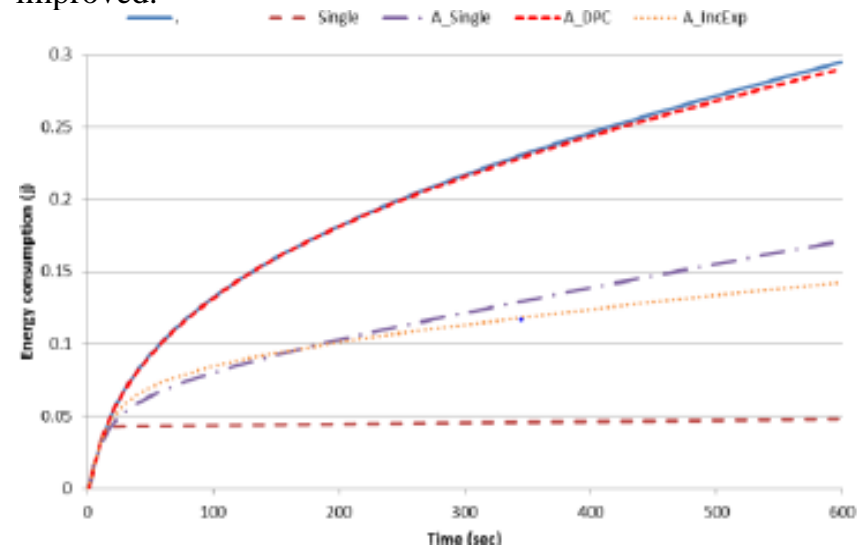

Fig. 4. Power utilization with Existing procedure and enhanced AEIAWSNHP.

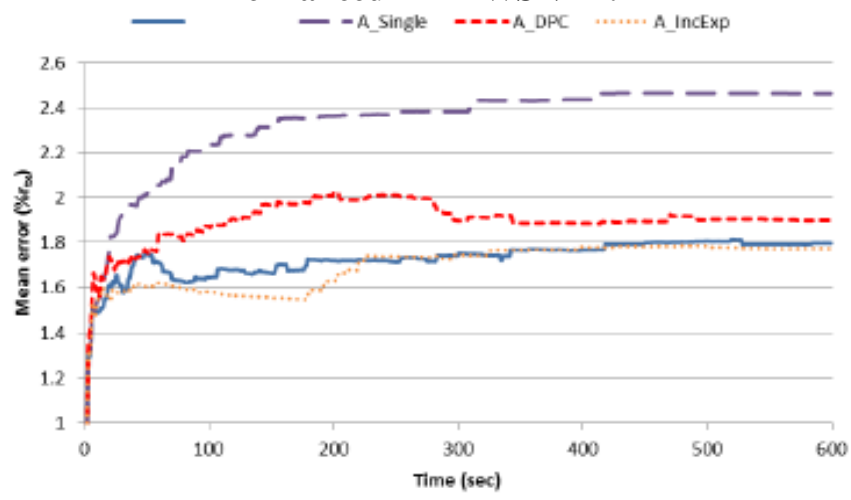

Fig. 5. Average fault as a communication limit ratio with the existing procedure and enhanced AEIAWSNHP.

\section{iv.Efficiency assessment}

TABLE 1 represent efficiency assessment amount the existing algorithm and the enhanced AEIAWSNHP procedure and the innovative method. Average fault with the power utilization are note down at the completion of execution. Enhanced AEIAWSNHP procedure effort better output in conditions of significant fault and power utilization. It permits Enhanced AEIAWSNHP to drop the power utilization with no compromise the location assessment correctness.

TABLE 1: Efficiency assessment of innovative techniques

\begin{tabular}{|c|l|l|l|}
\hline Methods & $\begin{array}{l}\text { Average } \\
\text { fault } \\
\text { occurred }\end{array}$ & $\begin{array}{l}\text { Power } \\
\text { utilization } \\
\text { (j) }\end{array}$ & $\begin{array}{l}\text { power } \\
\text { enhancem } \\
\text {-ent }\end{array}$ \\
\hline AEIAWSNHP & $0.796 \%$ & .294 & - \\
\hline Distinct & $1.461 \%$ & .170 & $41 \%$ \\
\hline APM & $0.901 \%$ & .291 & $2.7 \%$ \\
\hline Inc_Exp & $0.772 \%$ & .142 & $50.6 \%$ \\
\hline
\end{tabular}




\section{CONCLUSION}

In order for the updated AEIAWSNHP to become a powerful energy-consumption algorithm, various techniques are used to reduce processing and transmission costs. These technologies can improve the energy consumption of AEIAWSNHP compared to other consecutive refinements. Identify algorithms without comprising the accuracy of the point estimate [16]. In this article, we examined three methods for improving the energy efficiency of AEIAWSNHP. These methods are the different valuation method, the active power control and the incremental and exponential demand. The simulation results show that the incremental and exponentially improved AEIAWSNHP method gave the best results in terms of power utilization and correctness.

\section{REFERENCES}

1. A. M. Abu-Mahfouz and G. P. Hancke, ns-2 extension to simulate localization system in wireless sensor networks, in IEEE AFRICON Conference, pp. 1-7,2011.

2. F. Yaghoubi and A. Abbasfar, Energy-efficient RSSI-based localization for wireless sensor networks, IEEE Commun., vol. 18, no. 6, pp. 973-976, (2014).

3. A M abu-Mahfouz and Gerhard P. Hancke ,ALWadHA localization Algorithm:Yet more energy efficient,IEEE access, Vol 5,pp-6661-6667,2017.

4. I. F. Akyildiz, W. Su, Y. Sankarasubramaniam, and E. Cayirci, A survey on sensor networks, IEEE Commun. Mag., vol. 40, no. 8, pp. 102-105, (2002).

5. A. M. Abu-Mahfouz, Accurate and efficient localisation in wireless sensor networks using a best-reference selection, Ph.D. thesis, Depatment of Electrical, Electronic and Computer Engineering, University of Pretoria, Pretoria, South Africa, (2011).

6. A. M. Abu-Mahfouz, T. Olwal, A. Kurien, J. L. Munda, and K. Djouani, Toward developing a distributed autonomous energy management system (DAEMS), in Proceedings of the IEEE AFRICON 2015 Conference on Green Innovation for African Renaissancce, (2015), pp. $1-6$.

7. A. Alvi, S. Bouk, S. Ahmed, and M. Yaqub, Best-mac: Bitmap-assisted efficient and scalable tdma-based wsn mac protocol for smart cities, IEEE Access, vol. 4, no. 1, pp. 312-322, (2016).

8. A. M. Abu-Mahfouz, Y. Hamam, P. R. Page, and K. Djouani,Real-time dynamic hydraulic model for potable water loss reduction, Procedia Eng., vol. 154, no. 7, pp. 99-106, (2016).

9. M. Usman, A. A. Gebremariam, U. Raza, and F. Granelli, A Software-Defined Device-to-Device Communication Architecture for Public Safety Applications in 5G Networks, IEEE Access, vol. 3, no. 9, pp. 1649-1654, (2015).

10. A. Srinivasan and J. Wu, Wireless Sensor Networks (WSNs): Secure Localization, in Encyclopedia of Wireless and Mobile Communications, Second Edi., Borko Furht, Ed. CRC Press, (2013), pp. 2343-2351.

11. K. Biswas, V. Muthukkumarasamy, X. W. Wu, and K. Singh, An Analytical Model for Lifetime Estimation of Wireless Sensor Networks, IEEE Communications Letters, vol. 19, no. 9. pp. 1584-1587, (2015).

12. V. Kaseva, T. D. Hamalainen, and M. Hannikainen, Range-free algorithm for energy-efficient indoor localization in Wireless Sensor Networks, in Proceedings of the IEEE Conference on Design and Architectures for Signal and Image Processing (DASIP), (2011), pp. $1-8$.

13. J. J. Robles, S. Tromer, M. Quiroga, and R. Lehnert, Enabling low-power localization for mobile sensor nodes, in Proceedings of the IEEE International Conference on Indoor Positioning and Indoor Navigation (IPIN), (2010), pp. 1-10.

14. T. Bui, P. Xu, N. Phan, W. Zhu, and G. Wu, An Accurate and Energy-Efficient Localization Algorithm for Wireless Sensor Networks, in Proceedings of the 83rd IEEE Vehicular Technology Conference, (2016), pp. 1-5

15. A. Ahmed and M. Ibrahim, Cluster-based energy-aware localization algorithm for Wireless Sensor Networks, in Proceedings of the 11th IEEE International Conference on Computer Engineering \& Systems, (2016), pp. 323-328.

16. A. Guidara, F. Derbel, and M. Ben Jemaa, Energy-efficient model for indoor localization process based on wireless sensor networks, in Proceedings of the 13th IEEE International Multi-Conference on Systems, Signals \& Devices (SSD), (2016), pp. 512-517.
17. A. M. Abu-Mahfouz and G. P. Hancke, An efficient distributed localisation algorithm for wireless sensor networks: Based on smart reference-selection method, Int. J. Sens. Networks, vol. 13, no. 2, pp. 94-111, (2013).

18. A. M. Abu-Mahfouz and G. P. Hancke, Evaluating ALWadHA for providing secure localisation for wireless sensor networks, in IEEE AFRICON Conference, (2013), pp. 501-505.

19. C. Tran-Xuan, V.-H. Vu, and I. K. I. Koo, Calibration mechanism for RSS based localization method in wireless sensor networks, in Proceedings of the 11th IEEE International Conference on Advanced Communication Technology, (2009), vol. 1, pp. 560-563.

20. S. Zhang, G. Li, W. Wei, and B. Yang, A Novel Iterative Multilateral Localization Algorithm for Wireless Sensor Networks, J. Networks, vol 5, no. 1, pp. 112-119, (2010).

21. A. M. Abu-Mahfouz, G. Hancke, and S. Isaac, Positioning system in wireless sensor networks using NS-2, Softw. Eng., vol. 2, no. 4, pp. 91-100, (2012)

22. A. M. Abu-Mahfouz, L. P. Steyn, S. J. Isaac, and G. P. Hancke, Multi-level Infrastructure of Interconnected Testbeds of Large-scale Wireless Sensor Networks (MI2T-WSN), in Proceedings of the International Conference on Wireless Networks - ICWN '12, (2012), pp. 445-450.

23. A. G. Dludla, A. M. Abu-Mahfouz, C. P. Kruger, and J. S. Isaac, Wireless sensor networks testbed: ASNTbed, in Proceeding of the IST-Africa 2013 Conference, (2013), pp. 1-10.

24. J. Yick, B. Mukherjee, and D. Ghosal, Wireless sensor network survey, Comput. Networks, vol. 52, no. 12, pp. 2292-2330, 2008.

\section{AUTHORS PROFILE}

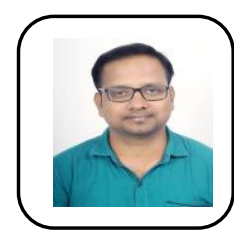

Chinmaya Kumar Nayak is a Research scholar of $\mathrm{PhD}$ in the Department of Computer science and Engineering,Veer Surendra Sai University of Technology (VSSUT), Burla, odisha, india. He published many papers in international seminars and journals. His research areas includes wireless Sensor Networks.

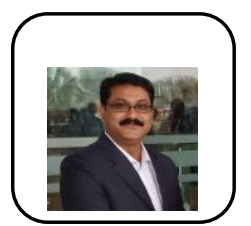

Satyabrata Das is working as an Associate professor in the Department of Information Technology,Veer Surendra Sai University of Technology (VSSUT), Burla, odisha, india. He published many papers in international seminars and journals.He is author of many books like Embedded System Development, Advanced Computer Architecture..etc. His research areas includes Distributed Systems, Cloud computing, Fault Tolerant Computing, Mobile Computing \& wireless Sensor Networks.. 